\title{
Clinical Manifestations of Influenza Caused by A/H1N1v Virus among Children and Teenagers Consulted in General Practice
}

\author{
A. Nitsch-Osuch ${ }^{1}$, E. Kuchar ${ }^{2}$, E. Gyrczuk ${ }^{1}$, A. Topczewska-Cabanek ${ }^{1}$, K. Zycinska ${ }^{1}$, K. Wardyn ${ }^{1}$ \\ ${ }^{1}$ Department of Family Medicine, Warsaw Medical University, Warsaw, Poland; ${ }^{2}$ Department of Pediatrics and Infectious Diseases, \\ Wroclaw Medical University, Wroclaw, Poland
}

\begin{abstract}
Background: Children and young adults are more susceptible to pandemic A/H1N1v infection than older people. There are some publications concerning the course of the pandemic influenza among pediatric population but mostly from hospital or from emergency units. There are very few observations of the course of pandemic influenza from primary care settings.

Objective: The aim of the study was to describe clinical manifestations of influenza caused by a pandemic strain $\mathrm{A} / \mathrm{H} 1 \mathrm{~N} 1 \mathrm{v}$ among children and teenagers younger than 14 years who were observed, diagnosed, and treated in general practice.

Material and methods: The observations were conducted among patients with symptoms of an acute respiratory tract infection in an urban area of Warsaw, Poland in November 2009. The inclusion criteria for performing a rapid influenza diagnostic test (RIDT) were established using the CDC definition of 'influenza-like illness' (ILI): fever $>38.7^{\circ} \mathrm{C}$ plus cough and/or sore throat in the absence of another known cause of illness. In patients who met ILI criteria, nasopharyngeal swabs were taken for RIDT and RT-PCR.

Results: 433 patients were consulted by a general practitioner and reported symptoms of an acute respiratory tract infection, $128(30 \%)$ of them met inclusion criteria for ILI and were tested with RIDT: 31 children younger than 14 years and 97 adults. All 31 children suspected of ILI also were tested by RT-PCR. 20 children had a positive result of the rapid influenza test. For all children tested negative, RT-PCR was also negative, and out of 20 children with the presumptive diagnosis of influenza established after rapid influenza test, the diagnosis was confirmed by RT-PCR in 18; their median age was 6.6 years and ranged from 13 months to 14 years. The most common symptoms of influenza A/H1N1v were: high fever $\left(>39^{\circ} \mathrm{C}\right)$ - $96 \%$ of patients, dry cough - $86 \%$ of patients, malaise - $78 \%$ of patients, headache - $66 \%$ of patients, and diarrhea or vomiting - $28 \%$ of patients. Two children received treatment with oseltamivir (one boy with congenital heart defect and Down's syndrome and another with severe bronchial asthma). The duration of symptoms ranged from 1 to 13 days (mean of 6.6 days). No patients required hospitalization either due to primary influenza infection or secondary complications.
\end{abstract}

Conclusions: The course of influenza caused by virus $\mathrm{A} / \mathrm{H} 1 \mathrm{~N} 1 \mathrm{v}$ in children younger than 14 years observed in a primary care setting was mild and self-limited without the necessity of antiviral treatment in most cases. A rapid influenza diagnostic test is helpful in diagnosing pandemic influenza.

Key words: pandemic influenza, clinical course, primary care

\section{INTRODUCTION}

At the end of March 2009, a new influenza virus A $(\mathrm{H} 1 \mathrm{~N} 1 \mathrm{v})$ of porcine origin was isolated from two children from California presenting a flu-like clinical syndrome. This virus was initially disseminated in Mexico and USA and then worldwide. Eight weeks later, it reached 74 countries with almost 30000 cases and caused 145 deaths. The 2009 outbreak of novel influenza A H1N1v reached a pandemic status in June 11, 2009. As of May 30, 2009 more than 214 countries and overseas territories or communities reported laboratory confirmed cases of influenza A H1N1v, including over 18138 deaths [1].

In Poland in 2009 and until May 15, 2010 there were reported 2917 cases of confirmed A H1N1v influenza, including 734 cases of children younger than 14 years [2]. Transmissibility of pandemic H1N1v infection is substantially higher than that of seasonal flu. It is, however, less than that which had been seen in the 1918 influenza pandemic, and comparable with the 1957 pandemic [3]. The finding that $60 \%$ of patients were 18 year of age or younger suggests that children and adolescents may be more susceptible to H1N1v infection than older persons $[4,5]$. Children younger than 5 years are more likely to suffer more complications after contracting the disease [6]. Severe underlying health conditions and immune deficiency are also considered to be the main risk factors leading to complications and hospitalization for children with pandemic influenza $[6,7]$. There are some publications concerning the course of the pandemic influenza among pediatric population, but mostly from hospitals or emergency units $[6,7]$. There are very few observations of the course of pandemic influenza from the primary care settings. Therefore, the aim of the present study was to describe clinical manifestations of 
influenza caused by a pandemic strain A H1N1v among children and teenagers younger than 14 years observed, diagnosed and treated in a general practice in an urban area of Warsaw in Poland.

\section{Material And Methods}

The study was performed in accordance with the Declaration of Helsinki for Human Research and was approved by a local Ethics Committee.

The observations of were conducted among patients with symptoms of an acute respiratory tract infection in general practice in Warsaw, Poland in November 2009. The inclusion criteria for performing a rapid influenza diagnostic test (RIDT; BD Directigen ${ }^{\text {TM }}$ EZ Flu A+B; Becton, Dickinson and Company, Sparks, MD, USA) had to meet the CDC definition of influenza-like illness' (ILI): fever $>38.7^{\circ} \mathrm{C}$ plus cough and/or sore throat in the absence of another known cause of illness. In patients who met the ILI criteria nasopharyngeal swabs were taken by trained personnel, using a special artificial stick (ending with viscose). The specimens were processed on-site at the general practitioner's office according to the manufacturer's recommendations. The nasopharyngeal swab method was chosen to assure the highest combination of sensitivity and specificity of RIDT. Positive and negative test results of RIDT were determined with the use of a visual key provided with the test kits. All patients enrolled for the RIDT also provided an additional nasopharyngeal swab specimen for a RT-PCR test, which was conducted within $24 \mathrm{~h}$ after it had arrived at the Voivoidship Sanitary-Epidemiological Station in Warsaw.

\section{RESULTS}

Four hundred thirty three patients were admitted by a general practitioner and reported symptoms of an acute respiratory tract infection, $128(30 \%)$ of them met inclusion criteria for ILI and had performed RIDT: 31 children younger than 14 years and 97 adults. Twenty children and teenagers, younger than 14 years old had a positive result of rapid influenza test, 11 children had a negative rapid influenza test. All 31 children suspected for ILI had also RT-PCR. For all children with a negative result for rapid influenza test, RT-PCR was also negative. Therefore, presumptive diagnosis of influenza was established in those 20 children positive for RIDT; the final diagnosis was confirmed in 18 young patients.

The median age of a child with pandemic influenza confirmed by RT-PCR was 6.6 years (range from 13 months to 14 years), and $61 \%$ of children with pandemic influenza were older than 5 years. Fourteen (78\%) of the 18 children who came down with confirmed influenza A H1N1v, were previously healthy, 4 $(22 \%)$ had had underlying health condition: two of them were diagnosed with bronchial asthma (a 3 years old boy and a 4 years old girl), a 4 -year-old boy had nephrotic syndrome and another boy aged 1.5 had a congenital heart defect (VSD) and Down's syndrome.

The most common symptoms of influenza A $\mathrm{H} 1 \mathrm{~N} 1 \mathrm{v}$ were: high fever $\left(>39^{\circ} \mathrm{C}\right)-96 \%$ of patients, dry cough $-86 \%$ of patients, malaise $-78 \%$ of patients, headache - $66 \%$ of patients, diarrhea or vomiting - $28 \%$ of patients. Two children received treatment with oseltamivir: the boy with congenital heart defect and Down's syndrome and the boy with severe bronchial asthma. Oseltamivir was given by 5 days, starting within the first $48 \mathrm{~h}$ of disease and was well tolerated. The child with a nephrotic syndrome and the other child with bronchial asthma were not treated with oseltamivir because of the late diagnosis and a mild disease course. Here, symptomatic home treatment sufficed.

Duration of symptoms ranged from 1 to 13 days (mean 6.6 days). Neither of the two children treated with oseltamivir revealed complications of influenza A H1N1v. Among 16 children treated symptomatically two developed complications and required antibiotic therapy due to the secondary infections (pneumonia in a 3 -year-old girl and otitis media in a 2 -year-old boy). No patients required hospitalization due neither to the primary influenza infection nor secondary complications.

\section{Discussion}

It is worth emphasizing that the present study has focused on children patients treated in the setting of primary care in contrast to other publications on pandemic influenza A H1N1v mostly devoted to hospitalized patients or patients from pediatric hospital emergency units [6-9]. Our present observations provide proof of a mild course of pandemic influenza among children without severe underlying diseases. In our study sample, most of infected with influenza A H1N1v children were older than 5 years, which is rather typical for pandemic influenza. Some other authors have also observed that children with pandemic influenza are slightly older than those with seasonal influenza (6 vs. 3 years) $[7,11]$.

The majority of cases of the 2009 pandemic influenza in children have been mild. However, severe disease does occur in children (especially younger than 5 years), including neurological or pulmonary complications, hospitalizations, or even deaths $[6,10]$. The risk factors for the 2009 influenza A H1N1v in pediatric population, the clinical course, and severity and complications, e.g., frequent gastrointestinal symptoms, have been similar to seasonal influenza [6-8]; these observations were confirmed in the present study, where $28 \%$ of sick children had gastrointestinal symptoms.

In the majority of cases, children with influenza, but with no signs of clinical severity or complications, do not require antiviral treatment. In the present study, although the results of RIDT tests were positive, such treatment was not administrated, which is compatible with current recommendations [12]. However, two of our children with underlying health problems, such as Down's syndrome and heart defect and severe asthma received oseltamivir. The other two, with nephrotic syndrome and bronchial asthma, would likely have received this treatment if they had been tested and diagnosed for influenza early enough. 
It should be stressed that physician's awareness of a rapid diagnosis of influenza in the primary care setting is important because it may significantly reduce the number of laboratory tests and radiographs ordered, along with the associated costs, and the number of antibiotics prescribed; allowing for more rational use of antivirals [13, 14]. This awareness would also allow testing in resource-limited settings, where equipment, reagents, and highly trained laboratory personnel are not always available. Rapid point-of-care diagnostic tests that can be performed within 15 min provide a significant time advantage over other laboratory-intensive influenza testing methods. The performance of rapid tests in detecting seasonal influenza $A$ and $B$ has been reported in numerous studies [13, 14]. Rapid tests have been shown to have lower accuracy than the reference tests of viral culture and reverse transcriptase polymerase chain reaction (RT-PCR), with reported sensitivity ranging from 27 to $90 \%$ and specificity $86-100 \%[15,16]$.

We conclude that the course of influenza caused by influenza virus A H1N1v in children younger than 14 years observed, diagnosed and treated in a primary care setting is mild and self limited, without the necessity of antiviral treatment in most cases. We also conclude that rapid influenza diagnostic tests are helpful in diagnosing pandemic influenza.

Acknowledgments: Supported in part by a grant from the Academy of Economics and Humanities in Lodz (no 2/2009).

Conflicts of interest: No conflicts of interests were declared by the authors in relation to this article.

\section{REFERENCES}

1. Pandemic (H1N1) 2009 - update 104. www.who.int/csr/don/2010_06_11/en/index

2. Reports on cases of infectious diseases and poisonings in Poland. National Institute of Public Health. www.pzh.gov.pl/oldpage/epimeld/index

3. Frazer C, Donnelly CA, Cuchemez S, Hanage WP, Van Kekrhove MD. Hollingsworth TD. Pandemic potential of a strain A (H1N1); early findings. Science 2009; 324: 1557-61.

4. Dawood FS, Jain S, Finelli L, Shaw MW, Lindstrom S, Garten RJ, Gubareva LV, Xu X, Bridges CB, Uyeki TM and Novel Swine-Origin Influenza A (H1N1) Virus Investigation Team. Emergence of a novel swine-origin influenza A (H1N1) virus in humans. N Engl J Med 2009; 360: 2605-15.

5. Mermel IA. Swine-origin influenza virus in young age groups. Lancet 2009: 373: 2108-9.

6. Lee HY, Wu Chang T, Kin TY, Chiu CH. 2009 pandemic influenza H1N1: Paediatric perspectives. Ann Acad Med Singapore 2010; 39: 333-335.
7. Plessa E, Diakakis P, Gardelis J, Thirios A, Kolesi P, Falagas ME. Clinical features, risk factors and complications among pediatric patients with pandemic influenza $A$ (H1N1). Clin Pediatr (Phila) 2010; 1: 23-6.

8. Gordon A, Saborío S, Videa E, López R, Kuan G, Balmaseda A, Harris E. Clinical attack rate and presentation of pandemic H1N1 influenza versus seasonal influenza A and $\mathrm{B}$ in a pediatric cohort in Nicaragua. Clin Infect Dis 2010; 50(11): 1462-7.

9. Saha A, Jha N, Dubey NK, Gupta VK, Kalaivani M. Swine-origin influenza A (H1N1) in Indian children. Ann Trop Paediatr 2010; 30(1): 51-5.

10. Halasa NB. Update on the 2009 pandemic influenza A H1N1 in children. Curr Opin Pediatr 2010; 22(1): 83-7.

11. O'Riordan S, Barton M, Yau Y, Read SE, Allen U, Tran D. Risk factors and outcomes among children admitted to hospital with pandemic H1N1 influenza. CMAJ 2010; 182(1): 39-44.

12. Mares J, Rodrigo C, Moreno-Perez D, Cilleruelo MJ, Barrio F, Bunuel JC, Gonzalez MP, Gonzalez de Dios J, Aparicio M, Arístegui J, Hernandez-Sampelayo T. Recommendations for the management of influenza in pediatrics (2009-2010). An Pediatr (Barc) 2010; 2: 235-8.

13. Bonner A, Monroe K, Talley L, Klasner A, Kimberlin D. Impact of the rapid diagnosis of influenza on physician decision-making and patient management in the pediatric emergency department: Results of a randomized, perspective, controlled trail. Pediatrics 2003; 112(2): 362-7.

14. de La Rocque F, Lecuyer A, Wollner C, d'Athis P, Pecking $\mathrm{M}$, Thollot $\mathrm{F}$, Cohen $\mathrm{R}$. Impact of influenza rapid diagnostic tests (IRDT) on the diagnosis of influenza and on the management of influenza in children in ambulatory pediatric setting. Arch Pediatr 2009; 16(3): 288-93.

15. Ginocchio CC, Zhang F, Manji R, Arora S, Bornfreund M, Falk L, Lotlikar M, Kowerska M, Becker G, Korologos D, de Geronimo M, Crawford JM. Evaluation of multiple test methods for the detection of the novel 2009 influenza A (H1N1) during the New York City outbreak. J Clin Virol 2009; 45: 191-5.

16. Performance of rapid influenza diagnostic tests during two school outbreaks of 2009 pandemic influenza A (H1N1) virus infection - Connecticut, 2009. MMWR CDC Surveillance Summaries 2009; 58: 1029-32.

\author{
Address for correspondence: \\ Aneta Nitsch-Osuch \\ Department of Family Medicine \\ Warsaw Medical University \\ $1 \mathrm{~A}$ Banacha St. \\ 02-097 Warsaw \\ Poland \\ Phone: +48 225992190 \\ Fax: +48 225992178 \\ E-mail: anitsch@amwaw.edu.pl
}

